\title{
Tratamiento antimicrobiano en niños con neutropenia febril de alto riesgo
}

\author{
ELIANA MUÑOZ B. ${ }^{1}$, JUAN CRISTÓBAL OSSA A. ${ }^{1}$, \\ MILENA VILLARROEL C. ${ }^{2}$, MARÍA ELENA SANTOLAYA DP. ${ }^{1,3}$ \\ 1. Becado de Pediatría, Departamento de Pediatría, Campus Oriente, Facultad de Medicina, Universidad de Chile. \\ 2. Unidad de Oncología, Hospital Luis Calvo Mackenna. \\ 3. Unidad de Infectología, Hospital Luis Calvo Mackenna.
}

\begin{abstract}
Antibiotic treatment in children with High Risk Febrile Neutropenia

Background: In children with cancer and high risk febrile neutropenia (HRFN), the initial empirical treatment used in our hospital for 5 years includes the association of Cloxacillin + Ceftazidime + Amikacin. There is no chilean literature that reviews the effectiveness of this therapy. Objective: Evaluate the clinical and microbiological effectiveness of this associated therapy in children with HRFN. Method: A prospectivedescriptive study evaluating children with HRFN admitted at Hospital Luis Calvo Mackenna between January 2005-August 2006. Results: 100 HRFN episodes were evaluated. In $48 \%$ of cases, the antimicrobial treatment was considered effective, whereas in $52 \%$ of episodes the therapy required modifications ( $15 \%$ cases within the first 72 hours). The most frequent diagnoses were fever without clinical focus $(51 \%)$ and sepsis (13\%). 36\% had microbiological identification and the most frequently isolated bacteria were Escherichia coli (9\%) and Staphylococcus aureus (9\%). Conclusions: A favorable answer with the initial empirical therapy was obtained for $48 \%$ of cases; meanwhile in the remaining episodes, $28 \%$ required antibiotics modifications without justification. This fact remarks the importance of following the established guidelines for antimicrobial treatment modification in these patients.

(Key words: Cancer, febrile neutropenia, antibiotics, oncology)

Rev Chil Pediatr 2008; 79 (4): 381-387
\end{abstract}

\section{RESUMEN}

Introducción: En los niños con cáncer y netropenia febril (NF) de alto riesgo, se utiliza en nuestro centro hace 5 años, como esquema empírico inicial, la asociación de ceftazidima-amikacina-cloxacilina. No hay literatura nacional que analice la eficacia de este esquema. Objetivo: Evaluar la eficacia clínica y microbiológica de la asociación de ceftazidima, amikacina y cloxacilina en niños con NF de alto riesgo. Método: Protocolo descriptivo, prospectivo. Evaluar niños con NF de alto riesgo hospitalizados en el HLCM entre enero 2005

Trabajo recibido el 14 de junio de 2007, devuelto para corregir el 11 de julio de 2007, segunda versión el 12 de diciembre de 2007, aceptado para publicación 02 de abril de 2008.

Financiamiento parcial: Proyecto Fondecyt 1040907

Correspondencia a:

Eliana Muñoz B.

E-mail: elianamunozb@gmail.com 
y agosto 2006. Resultados: 100 episodios de NF de alto riesgo. El tratamiento antimicrobiano fue considerado eficaz en $48 \%$ de los casos, y requirió ajustes en $52 \%$ de los casos. En 13\% se cambia tratamiento sin justificación y en $15 \%$ se realiza antes de 72 horas de iniciado el tratamiento antimicrobiano. Foco más frecuente fue: ausencia de foco clínico 51\%, presentando sepsis $13 \%$ de los niños. Hubo identificación microbiológica en 36\%, microorganismos más frecuentemente aislados Echerichia coli (9\%) y Staphylococcus aureus (9\%). Conclusiones: Se obtuvo una respuesta favorable de $48 \%$ con el esquema antimicrobiano empírico inicial, de el $52 \%$ restante, en un $28 \%$ se efectúan cambios de antibióticos no justificados, esto recalca la importancia de seguir las pautas ya establecidas para cambio de esquema antimicrobiano en estos pacientes.

(Palabras clave: Neutropenia febril, cáncer, oncología, antimicrobianos).

Rev Chil Pediatr 2008; 79 (4): 381-387

\section{Introducción}

En niños con cáncer las infecciones son una de las más frecuentes y serias complicaciones de la quimioterapia, constituyendo una importante causa de morbimortalidad. Los procesos infecciosos se ven favorecidos por la alteración de los mecanismos naturales de barrera producto de la mucositis, la utilización de catéteres venosos centrales y las múltiples punciones, así como por la disminución de la inmunidad inducida tanto por la patología de base como por el uso de agentes quimioterapéuticos, los cuales como efecto secundario producen neutropenia ${ }^{1-3}$.

La presencia de fiebre en el contexto de neutropenia severa se debe en la mayoría de los casos a infecciones bacterianas. La severidad y duración de la neutropenia se relacionan directamente con la probabilidad de adquirir estas infecciones, aumentando significativamente con un recuento absoluto de neutrófilos (RAN) menor de $500 / \mathrm{mm}^{3}$, siendo máximo con un recuento menor a $100 / \mathrm{mm}^{3} 4,5$.

El manejo terapéutico habitual del niño con cáncer, neutropenia severa y fiebre estuvo por muchos años basado en una hospitalización precoz e inicio de tratamiento antimicrobiano empírico, de amplio espectro, cuya duración dependía de la resolución del cuadro febril y la recuperación medular ${ }^{6}$. Esta estrategia de manejo agresiva para todos los pacientes con neutropenia febril (NF), es adecuada para un grupo de ellos, pero inapropiada para un importante número de pacientes, considerando que las hospitalizaciones prolongadas y el uso de antimicrobianos de amplio espectro conlleva numerosos efectos adversos como es la toxici- dad propia de los antimicrobianos, desarrollo de cepas resistentes, mayor riesgo de infecciones nosocomiales y de infecciones fúngicas y las repercusiones sobre la calidad de vida de estos pacientes y sus familias ${ }^{7-10}$.

En los últimos años se ha puesto énfasis en categorizar a los pacientes con NF en grupos de alto y bajo riesgo de desarrollar infección bacteriana invasora (IBI). En nuestro centro se realiza esta clasificación validada en un estudio multicéntrico, realizado por el comité de enfermedades infecciosas del PINDA, en seis hospitales del Área Metropolitana ${ }^{8,11}$, en base a cinco variables medidas al momento del ingreso: tipo de cáncer, presión arterial, intervalo entre el término del último ciclo de quimioterapia y el inicio de la fiebre, nivel sérico de proteína $\mathrm{C}$ reactiva $(\mathrm{PCR})$ y recuento de plaquetas.

Se considerará que un niño cursa con un episodio de alto riesgo si hay presencia de dos o más de los factores de riesgo considerados o uno de los siguientes como factor único: PCR $>90 \mathrm{mg} / \mathrm{L}$, hipotensión, recaída de leucemia, leucemia no linfoblástica, linfoma no Hodgkin y neuroblastoma tipo $\mathrm{IV}^{8,11}$.

En la literatura se describen diversos tratamientos antimicrobianos empíricos basados en la microbiología aislada en este tipo de pacientes y en la epidemiología local de cada centro. El uso de un antibiótico $\beta$ lactámico más un aminoglicósido es una de las combinaciones más usadas, porque entrega cobertura de amplio espectro particularmente a microorganismos Gram negativos ${ }^{2}$. La eficacia de estos regímenes varía entre $50-90 \%{ }^{16}$. Existe controversia a nivel mundial en cuanto al uso y efectividad de la monoterapia con cefalosporinas de tercera o 
cuarta generación o con carbapenémicos versus la terapia combinada con un aminoglicósido $^{12,13}$.

En el Hospital Dr. Luis Calvo Mackenna (HLCM) desde el año 2002 se ha utilizado como esquema empírico inicial en pacientes de alto riesgo, la asociación de ceftazidimaamikacina-cloxacilina, basado en la identificación microbiológica encontrada, que corresponde con mayor frecuencia a Escherichia coli, Staphylococcus aureus, Staphylococcus coagulasa negativa, Klebsiella pneumoniae y Pseudomonas aeruginosa ${ }^{14}$.

No hay literatura nacional que analice la eficacia clínica y microbiológica de este esquema en los pacientes de alto riesgo y si es o no suficiente o excesivo como esquema antimicrobiano. Existen datos no publicados, generados por un estudio retrospectivo que evaluó los distintos esquemas antimicrobianos empíricos usados en cinco centros del Área Metropolitana, que evidenció que un $57 \%$ de los episodios de NF terminaron su tratamiento con éxito clínico o bacteriológico y que en un $23 \%$ de los episodios se efectuó un cambio terapéutico ${ }^{15}$. En base a estos antecedentes se realizó este estudio prospectivo en el HLCM, para analizar la eficacia clínica y microbiológica del tratamiento triasociado empírico inicial en niños con NF de alto riesgo.

\section{Pacientes y Métodos}

Diseño general: Protocolo prospectivo, descriptivo, desarrollado en las unidades de hematooncología e infectología del Hospital Dr. Luis Calvo Mackenna, Departamento Pediatría, Facultad de Medicina, Universidad de Chile, apoyado por el proyecto FONDECYT 1040907 y aprobado por el Comité de ética científico pediátrico del Servicio de Salud Metropolitano Oriente. Se incluyó a pacientes $\leq 20$ años con cáncer en tratamiento quimioterápico, que consultaron por neutropenia severa (RAN $\leq 500 /$ $\left.\mathrm{mm}^{3}\right)$ y fiebre $\left(\mathrm{t}^{\circ}\right.$ axilar $\geq 38,5{ }^{\circ} \mathrm{C}$ en una toma, o $\geq 38^{\circ} \mathrm{C}$ objetivada en 2 ocasiones, separadas por una hora) durante el período de tiempo necesario para recolectar 100 episodios de NF de alto riesgo. Al ingreso se realizó predicción de riesgo de IBI de acuerdo al modelo de predicción de riesgo creado y validado por el Comité de Infectología del PINDA ${ }^{11,19}$. El modelo incluye la evaluación de las siguientes variables: tipo de cáncer, número de días desde la última quimioterapia, presión arterial, PCR cuantitativa y recuento de plaquetas. Se consideró que un niño cursaba con un episodio de alto riesgo de IBI si presentaba dos o más factores de riesgo, o uno de los siguientes como factor único: PCR $>90 \mathrm{mg} / \mathrm{L}$, hipotensión, recaída de leucemia, leucemia no linfoblástica, linfoma no Hodgkin, o neuroblastoma tipo IV.

Los pacientes que no cumplieron con los criterios de alto riesgo de IBI fueron excluidos del estudio al igual que aquellos $>$ de 20 años y los sometidos a trasplante de precursores hematopoyéticos. Los pacientes con episodio de alto riesgo de IBI fueron invitados a participar, e ingresaron a nuestro protocolo luego de que sus padres o representantes legales aprobaran y firmaran el consentimiento informado, y los mismos niños en caso de ser mayores de 12 años. Todos los niños con episodios de alto riesgo de IBI fueron evaluados al ingreso y durante su hospitalización con el objetivo de analizar la eficacia clínica y microbiológica del tratamiento antimicrobiano empírico utilizado. Al término de la hospitalización, un investigador analizó los episodios a fin de determinar la evolución y el tratamiento utilizado.

Evaluación y seguimiento: Al ingreso de cada paciente se registraron las siguientes variables: demográficas: edad, género; clínicas: estado general, temperatura axilar, presión arterial; laboratorio: RAN, PCR cuantitativa, recuento de plaquetas, hemocultivos central y periférico, orina completa, cultivo de orina y de otros sitios estériles de acuerdo a la orientación clínica, y radiografía de tórax. Se realizó un seguimiento clínico consistente en control diario de temperatura, estado general y presión arterial, hasta completar 48 horas afebril. Se evaluó recuento de neutrófilos al ingreso, los días 2 y 3 de evolución, y luego cada dos días hasta que el RAN fuera $>500 / \mathrm{mm}^{3}$; PCR cuantitativa al momento del ingreso, al día 2 y 3 de evolución, y luego cada dos días hasta obtener 2 valores consecutivos $<40 \mathrm{mg} / \mathrm{L}$; control de hemocultivos $\mathrm{u}$ otros cultivos $(+)$ a las 72 horas luego de todo 
cultivo que resultara positivo en el día del ingreso del paciente al protocolo. En caso de haber un cultivo positivo en el día 3 , se repitió la toma de muestra para nuevo cultivo cada tres días hasta evidenciar su negativización. Se consignó cada cambio de esquema antimicrobiano y la razón clínica o microbiológica para realizarlo.

\section{Definiciones}

Evolución favorable: Estabilidad hemodinámica, ausencia de fiebre, ausencia de nuevo foco infeccioso no cubierto, curva de PCR en descenso.

Evolución desfavorable: Que no cumpla las condiciones de evolución favorable.

Eficacia clínica y/o microbiológica: Aquellos episodios que experimentaron evolución favorable y/o negativización de cultivos, con el esquema antimicrobiano inicial o en aquellos que se restringió el espectro de acción.

Criterios de cambio tratamiento antibiótico: Tras $72 \mathrm{~h}$ con terapia antimicrobiana inicial y con evolución desfavorable, o por hallazgos microbiológicos que lo justifiquen.

Análisis estadístico: Se realizó análisis crítico de fichas clínicas y de los resultados obtenidos, mediante la utilización de variables de tendencia central, según el tipo de variable analizada.

\section{Resultados}

Se analizaron un total de 100 episodios de NF de alto riesgo entre enero 2005 y agosto 2006, en un total de 61 pacientes. La edad promedio fue de 9 años (rango 1-20 años), 34 hombres $(55 \%)$, siendo $36 \%$ portadores de leucemia linfoblástica aguda (tabla 1). Los focos clínicos más frecuentemente encontrados fueron: ausencia de foco clínico $51 \%$, respiratorio $27 \%$, enteral $9 \%$, mucocutáneo $8 \%$, urinario $3 \%$, infección de catéter venoso central $2 \%$ (figura 1). Se diagnosticó sepsis en 13\% de los episodios. Hubo identificación microbiológica en 36\% de los casos, siendo los microorganismos más frecuentemente aislados Escherichia coli (9\%) y Staphylococcus aureus (9\%). En cuanto a la susceptibilidad a antimicrobianos, destaca la ausencia de $S$. aureus oxacilina resisten- te; y la casi totalidad (5/6) de Staphylococcus coagulasa (-) oxacilina resistente. Se obtuvieron 5 IFI virales positivas de aspirado nasofaríngeo: 3 adenovirus y 2 virus respiratorios sincicial, un caso de aislamiento de Candida albicans en orina, y uno de Cryptosporidium parvum en deposiciones (tabla 2).

En cuanto al tratamiento antimicrobiano, éste fue considerado eficaz (se mantuvo o se redujo) en $48 \%$ de los casos, y se ajustó ampliando la cobertura en $52 \%$. El cambio de esquema antimicrobiano se realizó en $6 \%$ de los casos por motivos microbiológicos (resistencia in vitro o ausencia de cobertura del microorganismo identificado). Del $46 \%$ restante, en un $15 \%$ de los episodios se realizó el cambio de tratamiento antes de lo recomendado (72 horas de inicio terapia antimicrobiana) y en $13 \%$ no hubo una razón justificada en la ficha clínica (figura 2). Los antibióticos indicados más frecuentemente en estos casos fueron vancomicina (48\%), ciprofloxacina (28\%) y metronidazol (14\%).

Tabla 1. Características generales al ingreso de 61 niños con 100 episodios de neutropenia febril de alto riesgo

\begin{tabular}{llr}
\hline Edad (promedio) & 9 años (rango 1 - 20 años) \\
Género & Masculino 34 (55\%) & \\
& Femenino 27 (45\%) & \\
Enfermedad oncológica & Leucemia linfoblástica aguda $36 \%$ \\
de base & Osteosarcoma & $16 \%$ \\
& Leucemia no linfoblástica & $15 \%$ \\
& Sarcoma de partes blandas & $7 \%$ \\
& Neuroblastoma & $7 \%$ \\
& Otros & $19 \%$ \\
Horas de fiebre previo & $10(1-96)$ & \\
al ingreso: $X$ (rango) & & \\
& & \\
& &
\end{tabular}

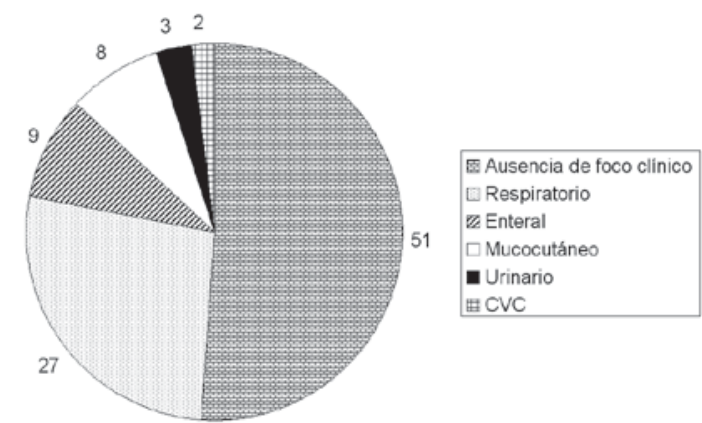

Figura 1. Focos clínicos en 100 episodios de neutropenia febril de alto riesgo. 
Tabla 2. Microorganismos identificados en 100 episodios de neutropenia febril de alto riesgo

\begin{tabular}{|c|c|c|c|c|c|c|c|}
\hline Microorganismo & Sangre & Catéter & $\begin{array}{l}\text { Sangre } \\
\text { y catéter }\end{array}$ & Orina & Bronquio & Otros & Total \\
\hline Escherichia coli MS & 2 & 1 & 2 & 3 & & & 8 \\
\hline Escherichia coliMR & & & & 1 & & & 1 \\
\hline Staphylococcus aureus MS & & 4 & 1 & & 1 & 3 & 9 \\
\hline Staphylococcus aureus MR & & & & & & & 0 \\
\hline Staphylococcus coagulasa (-) MS & & & 1 & & & & 1 \\
\hline Staphylococcus coagulasa (-) MR & 4 & & 1 & & & & 5 \\
\hline Pseudomonas aeruginosa & & 1 & 1 & & & & 2 \\
\hline Klebsiella pneumoniae MR & 1 & & & & & & 1 \\
\hline Streptococcus viridans & 1 & & & & & & 1 \\
\hline Streptococcus pneumoniae & 1 & & & & & & 1 \\
\hline Candida albicans & & & & 1 & & & 1 \\
\hline Cryptosporidium parvum & & & & & & 1 & 1 \\
\hline Adenovirus & & & & & & 3 & 3 \\
\hline Virus respiratorio sincicial & & & & & & 2 & 2 \\
\hline Total & & & & & & & 36 \\
\hline
\end{tabular}

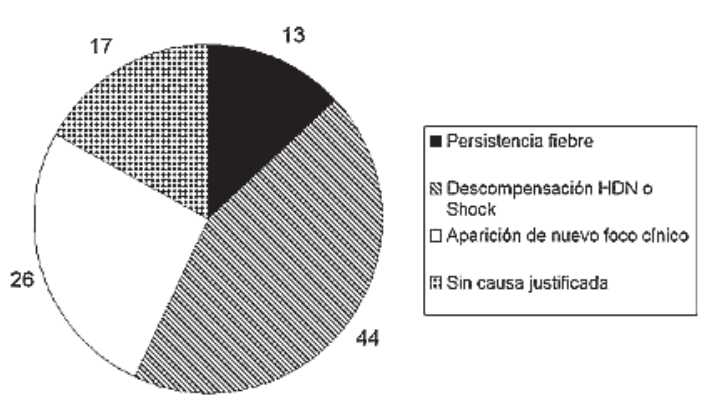

Figura 2. Motivos de cambio en esquema antimicrobiano.

La duración promedio del tratamiento antibiótico fue de 11 días (rango 2-42 días), 24\% de los episodios terminaron su tratamiento con antibióticos por vía oral, de los cuales el más frecuentemente utilizado fue cefuroximo.

Se indicó tratamiento antifúngico en 9 pacientes, con una duración promedio de tratamiento de 12 días (rango 4-21 días). Los motivos para esta indicación fueron fiebre persistente y PCR en aumento en 5 casos, lesiones pulmonares inespecíficas en 3 casos y determinación de galactomanano $(+)$ en 1 caso.

Durante el período de estudio fallecieron 4 pacientes, catalogados por el equipo de oncolo- gía/infectología como mortalidad relacionada al episodio de NF, 3 de ellos con hemocultivos positivos: $S$. aureus oxacilina sensible, E. coli multisensible más $K$. pneumoniae multirresistente, y $S$. coagulasa negativo oxacilina resistente más $E$. coli multisensible.

\section{Discusión}

El propósito de nuestro estudio consistió en analizar la eficacia clínica y microbiológica del tratamiento triasociado empírico inicial en niños con NF de alto riesgo, considerando las variables epidemiológicas, clínicas y microbiológicas involucradas. Destaca en nuestra serie de 100 episodios de NF de alto riesgo el hallazgo de microbiología positiva en cerca de $1 / 3$ de los episodios, lo que concuerda con literatura internacional y nacional previa ${ }^{12,14,16}$, asimismo los microorganismos más frecuentemente encontrados corresponden a E. coli y $S$. aureus ${ }^{11,12,14}$. Considerando que en $51 \%$ de los episodios no hay foco clínico evidente, asociado a microbiología positiva en $1 / 3$ de los episodios, se fundamenta la importancia de obtener cultivos sanguíneos y de otros líquidos orgánicos habitual- 
mente estériles a fin de obtener la etiología del proceso infeccioso y de esta forma racionalizar el tratamiento antimicrobiano.

En 52\% de los episodios se amplió el espectro de cobertura antimicrobiana, el antibiótico más frecuentemente empleado fue vancomicina (48\%), lo que llama la atención al correlacionar con la sensibilidad de la microbiología obtenida en la cual no hay $S$. aureus oxacilina resistente y sólo $5 S$. coagulasa (-) oxacilina resistente. Esto, sumado a que en $13 \%$ de estos pacientes se cambia tratamiento sin justificación y que en $15 \%$ se realiza antes de 72 horas de iniciado el tratamiento antimicrobiano, recalca la importancia de seguir las pautas ya establecidas para cambio de esquema antimicrobiano en estos pacientes $^{12-14}$, y el uso racional de vancomicina ${ }^{14}$.

Existe discrepancia respecto a estudios previos en cuanto al aislamiento de Pseudomonas aeruginosa como factor etiológico responsable de morbimortalidad en pacientes con NF de alto riesgo ${ }^{16,17}$. En nuestro estudio hubo aislamiento en 2 casos y no relacionados con los episodios fatales, lo que lleva a cuestionarse la necesidad de mantener como parte del esquema antimicrobiano empírico inicial el uso de ceftazidima. Cabe destacar el bajo porcentaje de mortalidad (4\%), considerando que $13 \%$ de los episodios presentaron sepsis durante la evolución, lo que refleja el adecuado manejo y tratamiento en esta condición.

Este estudio evidencia eficacia del esquema empírico inicial en niños con NF de alto riesgo en $48 \%$ de los episodios, en el $52 \%$ restante se amplia la cobertura antimicrobiana, con una real indicación de cambio en $37 \%$ de los episodios. Estos resultados son concordantes con un estudio chileno multicéntrico retrospectivo no publicado (PINDA 1998).

Nuestro estudio describe la conducta actual sobre uso y ajuste de terapia antimicrobiana en una población de niños con NF de alto riesgo. La Sociedad Chilena de Infectología, junto con la Sociedad Chilena de Hematología, el Programa Infantil Nacional de drogas antineoplásicas y el Programa adulto Nacional de drogas antineoplásicas aunaron sus esfuerzos, y publicaron el año 2005, un Consenso nacional sobre Manejo racional del paciente con cáncer, neutropenia y fiebre ${ }^{18}$, que entrega pautas de evalua- ción de la respuesta terapéutica e indicaciones de ajuste de terapia antimicrobiana en los episodios de NF.

Teniendo en consideración que el modelo descriptivo utilizado en nuestro estudio tiene limitaciones, y que se requieren ensayos comparativos para un mayor y mejor análisis de la eficacia del tratamiento antimicrobiano en este grupo de pacientes, consideramos que nuestros datos aportan a reconocer que cerca de un tercio de los ajustes de terapia antimicrobiana en nuestra población fueron hechos sin justificación adecuada, lo que pone de manifiesto la importancia de seguir las pautas establecidas para manejo y seguimiento de estos pacientes.

\section{Referencias}

1- Pizzo PA, Rubin M, Freifeld A, et al: The child with cancer and infection: I. Empiric therapy for fever and neutropenia, and preventive strategies. J Pediatr 1991; 119: 679-94.

2.- Pizzo PA, Rubin M, Freifeld A, Walsh TJ: The child with cancer and infection II. Nonbacterial infections. J Pediatr 1991; 119: 845-57.

3.- Riikonen P, Jalanko H, Hovi L, Saarinen UM: Fever and neutropenia in children with cancer: diagnostic parameters at presentation. Acta Paediatr 1993; 82: 271-5.

4.- Bodey GP, Buckley M, Sathe YS, Freireich EJ: Quantitative relationships between circulating leukocytes and infection in patients with acute leukemia. Ann Intern Med 1966; 64: 328-40.

5.- Tordecilla J, Campbell M, Joannon P: Neutropenia febril en niños con cáncer. Rev Chil Pediatr 1994; 65: 149-53.

6.- Pizzo PA, Rubin M, Freifeld A, Walsh TJ: The child with cancer and infection. I. Empiric therapy for fever and neutropenia, and preventive strategies. J Pediatr 1991; 119: 9-94.

7.- Anaissie E, Vadhan-Raj $S$ : Is it time to redefine the management of febrile neutropenia in cancer patients? Am J Med 1995; 98: 221-3

8.- Santolaya ME Álvarez AM, Avilés C, et al: Neutropenia febril en el niño con cáncer: conceptos actuales sobre criterios de riesgo y manejo selectivo. Rev Méd Chile 2001; 129: 1449-454.

9.- Rona E, Vargas L: Problemas psicológicos en la familia del niño con cáncer. Rev Chil Pediatr 1992; 63: 222-9

10.- Tordecilla J, Campbell M, Joannon P, Rodríguez N: Alta precoz de niños con cáncer y neutropenia febril. Rev Chil Ped 1994; 65: 260-3.

11.- Santolaya ME, Álvarez AM, Becker A, et al: Prospective, Multicenter Evaluation of Risk Factors Associated with Invasive Bacterial Infection in Children 
with Cancer, Neutropenia and Fever. J Clin Oncol 2001; 19: 3415-21.

12.- Huges W, Armstrong D, Bodey G, et al: Guidelines for the use of antimicrobial agents in neutropenic patients with cancer. Clin Infect Dis 2002; 34: 730-51.

13.- Tamura $K$, Imajo $K$, Akimaya $N$, et al: Randomized trial of Cefepime Monotherapy or Cefepime in Combination with Amikacin as Empirical Therapy for Febrile Neutropenia. Clin Infect Dis 2004; 39: 15-24.

14- Santolaya ME, Álvarez AM, Aviles $C$, et al: Tratamiento selectivo de los episodios de neutropenia febril en niños con cáncer. Comité de Infectología, Programa Infantil Nacional De Drogas Antineoplásicas (PINDA). Rev Chil Infect 2004; 21 (3): 213-22.

15.- Santolaya ME, Álvarez AM, Becker A, et al: Eficacia de los esquemas antimicrobianos empíricos actualmente en uso en el manejo de los episodios de neutropenia febril en niños con cáncer. Datos no publicados. Libro resumen XV Congreso chileno de Infectología, Santiago Agosto 1998.

16.- Miranda-Novales $M G$, Belmont $M$, Villasis $K$, et al: Empirical antimicrobial therapy in pediatric patients with neutropenia and fever. Risk Factors for Treatment Failure. Arch Med Res 1998; 29 (4): 331-5.

17.- Chatzinikalaou I, Abi-Said, Bodey GP, et al: Recent experience with Pseudomonas aeruginosa bacteremia in patients with cancer: retrospective analysis of 245 episodes. Arch Intern Med 2000; 160: 501-9.

18.- Santolaya ME, Rabagliatti, $R$, Bidart $T$, et al: Consenso nacional: Manejo racional del paciente con neutropenia y fiebre. Rev Chil Infectol 2005; 22: S79113.

19.- Hughes R, Armstrong B, Bodey K, et al: Guidelines for the use of antimicrobial agents in neutropenic patients whith cancer. Clin Infect Dis 2002; 34: 73051 . 